\title{
Una experiencia en el uso de metaversos para la enseñanza de la física mecánica en estudiantes de ingeniería
}

\author{
Jorge Augusto Jaramillo-Mujica ${ }^{a}$, Leonel Felipe Morales-Avella ${ }^{a} \&$ Diana Marcela Coy-Mondragón ${ }^{b}$ \\ a Ingeniería en Multimedia de la Facultad de Ingeniería, Universidad Militar Nueva Granada, Bogotá, Colombia.jorge.jaramillo@unimilitar.edu.co, \\ u1201081@unimilitar.edu.co

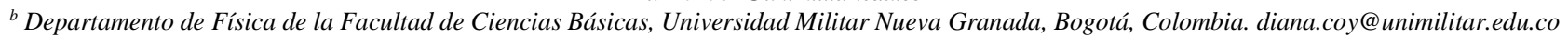

Recibido para revisar Marzo 2 de 2017, aceptado Mayo 9 de 2017, versión final Mayo 24 de 2017

\begin{abstract}
Resumen - Una de las tendencias en la aplicación de tecnologías emergentes en educación, son los mundos virtuales que llevados a contextos en Internet están prometiendo interesantes avances, de manera que se ha propuesto la idea de diseñar e implementar un entorno inmersivo 3D o metaverso, para incentivar el aprendizaje en Física Mecánica de estudiantes de ingeniería. Los resultados estadísticos en la pérdida de asignaturas en el área de las Ciencias Básicas en la Universidad Militar Nueva Granada, han oscilado entre el $40 \%$ y el $60 \%$, encendiendo las alarmas en los directivos de la Universidad. En este trabajo se describen las fases de desarrollo, conceptualización e implementación de los escenarios en la plataforma OpenSim, articulados desde un aula virtual en Moodle, presentando al final los resultados luego de haberse desarrollado un piloto a dos grupos de estudiantes de segundo semestre de la Facultad de Ingeniería, quienes estuvieron interactuando y participando de las actividades propuestas.
\end{abstract}

Palabras Clave - Metaverso, escenarios 3D, física mecánica, OpenSim.

\section{An experience using metaverses for teaching mechanical physics to engineering students}

\begin{abstract}
One of the trends in the application of emerging technologies in education are virtual worlds, that brought to the internet are promising interesting advances, so the idea of designing and implementing an immersive $3 \mathrm{D}$ environment or metaverse has been proposed to encourage learning in Physics Mechanics of engineering students. The statistical results in subject's failure in the Basic Sciences area at the Universidad Militar Nueva Granada, have fluctuated from $40 \%$ to $60 \%$, igniting the alarms in the University executives. This paper describes the stages of development, conceptualization and implementation of the scenarios in the OpenSim platform, articulated from a virtual classroom in Moodle, the results presented at the end were obtained by a test applied over two groups of students; they were taking their first year of engineering degree and participated in the proposed activities.
\end{abstract}

Keywords- Opensim, Virtual Environments, metaverse, physics.

\section{Introducción}

En el proceso de formación de Ingenieros, las áreas de las ciencias básicas conforman un pilar fundamental, ya que permiten el desarrollo de habilidades y competencias disciplinares a través de la observación, inferencia, comprobación, comparación, afianzamiento, entre otras y así lograr el acceso al conocimiento [1]. Lo anterior ha motivado a las instituciones educativas y sus docentes, la puesta en escena de diferentes metodologías de enseñanza y aprendizaje, las que se han visto afectadas de diversas maneras, a cuenta de los avances en las tecnologías de la información al punto de la utilización de diversas herramientas digitales [2], tratándose de desarrollar aprendizajes más efectivos para encontrar cuál apoya mejor los procesos educativos y así aportando de manera significativa la base de conocimiento de los modelos pedagógicos más adecuados [3,4].

Una situación preocupante en la Universidad Militar Nueva Granada, ha sido que el área de ciencias básicas en las carreras profesionales para la formación de ingenieros, ha tenido altos porcentajes de pérdida, encontrándose entre el $40 \%$ y el $60 \%$. En la Fig. 1, se presentan los datos estadísticos obtenidos del sistema de información académico "Open Reports", para el programa de ingeniería en multimedia, en donde se puede observar que la asignatura de Física Mecánica ha llegado a tener hasta el 70\% de estudiantes que la han reprobado. Esta situación ha encendido las alarmas en los directivos de la universidad, quienes están intentando buscar las posibles causas de este bajo rendimiento. Se han estado tomando algunas medidas buscando mejorar la situación, como el diseño de actividades en el aula virtual y el aumento en docencia directa en algunas asignaturas.

En las reuniones de decanos se ha propuesto romper los mitos en el proceso de enseñanza, usando la motivación como estrategia educativa, utilizar objetos virtuales de aprendizaje y diseñar proyectos de investigación orientados a la construcción de materiales didácticos. Es por esta razón que el presente proyecto ha buscado abordar esta problemática y así aportar algunos de estos recursos en beneficio de los procesos educativos.

En el 2008, la Academia Nacional de Ingeniería (NAE) identificó la realidad virtual como uno de los 14 grandes desafíos para el siglo XXI, siendo una prioridad tecnológica digna de ser desarrollada [5]. A esto se le puede sumar la metodología de estudio “aprender haciendo" propuesta por Schank, quién la incorporó en un modelo para la enseñanza y el aprendizaje llamado GBS (Goal Based Scenarios) para afrontar los problemas relacionados con los entornos de aprendizaje tradicionales, y en donde los estudiantes persiguen una meta practicando las habilidades necesarias para lograrla [6]. Es así como el "aprender haciendo" aplicado en ambientes tecnológicos basados en mundos virtuales, permiten y facilitan su aplicación, especialmente en contextos del e-learning [7].

Como citar este artículo: Jaramillo-Mujica, J.A., Morales-Avella, L.F. and Coy-Mondragón, D.M., Una experiencia en el uso de metaversos para la enseñanza de la Física Mecánica en estudiantes de ingeniería. Educación en Ingeniería, 12(24), pp. 20-30, Julio, 2017. 


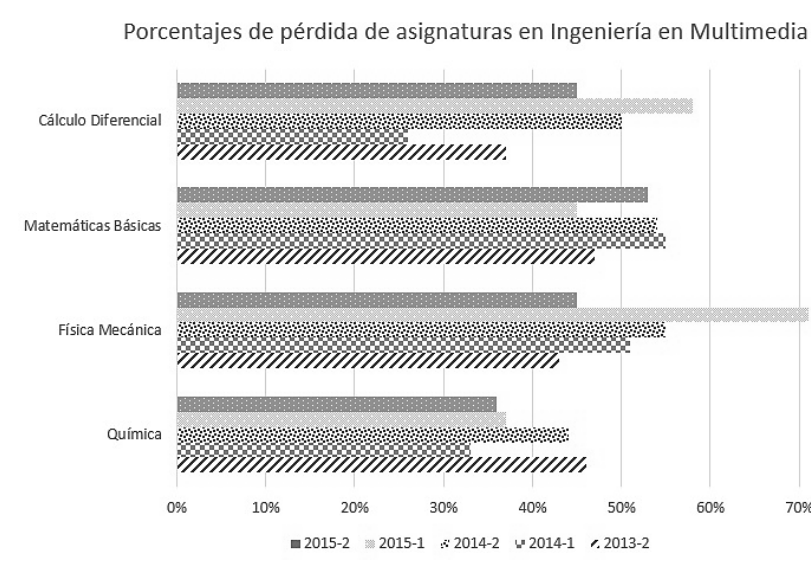

Figura 1. Asignaturas que más pierden los estudiantes del programa de Ingeniería en Multimedia de la Universidad Militar Nueva Granada. Fuente: Los autores.

A través de estos mundos virtuales soportados en internet, conocidos también como metaversos, es posible recrear escenarios reales o imaginarios en un computador, en donde los usuarios pueden interactuar a través de personajes virtuales llamados "avatares", dando la sensación de estar inmerso. Todo esto es posible gracias a la generación de imágenes en tiempo real, desplegadas en el área de visualización del usuario, en el mismo instante de interactuar y moverse en el escenario diseñado. Gilbert ha identificado cinco características esenciales que estos entornos deben tener [5]: Poseer una interfaz gráfica 3D con audio integrado, soportar la interacción de múltiples usuarios en forma remota, ser persistente manteniéndose funcional y operable constantemente aunque sus usuarios no estén conectados, ser inmersiva generando la sensación de realismo creando un sentido de presencia y por último, poder enfatizar en las actividades, objetivos y herramientas de creación de contenidos para personalizar en contexto y la experiencia. Castronova a parte de la interactividad y la persistencia, agrega otra característica relacionada con la corporeidad, donde los usuarios se representan por avatares con una altura, peso y características físicas en un espacio sometido a determinadas leyes físicas [8].

Con estos espacios se ha buscado proponer su aplicación a contextos educativos prácticamente desde su aparición, a partir de escenarios para aprendizajes alternativos a las tecnologías educativas $[9,10]$. Además, permiten crear cualquier tipo de contenido para e-learning, visitas virtuales, simulaciones o cualquier escenario que se pueda imaginar ya sea para reproducción local o distribuida [11].

Una de las plataformas que ha liderado el desarrollo de este tipo de escenarios ha sido SecondLife, creada en 2003 por Linden Lab ${ }^{\circledR}$. A raíz de sus exigentes requerimientos en hardware y ancho de banda, implicó el abandono de muchos usuarios, razón por la cual decidió liberar el código fuente de su visor bajo licenciamiento GPL en el 2007. En este mismo año nace OpenSim como un servidor de aplicaciones 3D, gracias al trabajo de ingeniería de reversa por desarrolladores independientes. Con este lanzamiento, se pone a disposición de toda la comunidad académica del mundo, una alternativa de código abierto para el desarrollo de contenidos y actividades, sin tener que pagar por sus servicios. Esto ha ayudado a impulsar en las universidades, el desarrollo de innumerables proyectos de investigación, facilitando todos los niveles de personalización deseados y permitiendo a los estudiantes a través de su interfaz tridimensional, encontrar espacios más entretenidos que aquellas plataformas de aprendizaje LMS (Learning Management Systems) $[9,11,7]$.

Actualmente se pueden encontrar una gran variedad de entornos virtuales 3D, donde muchos de estos escenarios buscan acercar al estudiante hacia el conocimiento en formas lúdicas [3]. Es así como en diversos estudios relacionados con el uso de metaversos aplicados a educación, se han centrado en temas como la teoría del electromagnetismo, programación de algoritmos, arqueología, patrimonio cultural y ayuda humanitaria, entre otras, y han basado su estrategia de motivación que va más allá que aquellos materiales de estudio convencionales puestos a disposición en plataformas LMS. Pero, ¿Qué es lo que hace interesante y atractivo a estos escenarios virtuales, comparados con entornos 2D web? [12].

Las siguientes son algunas de las características que estas plataformas ofrecen para dar la sensación de inmersión. Cargadores de escenas 3D, que permiten leer desde archivos especiales, los escenarios tridimensionales o los componentes que la forman, como sonidos, personajes, imágenes, entre otras. Las diversas formas de navegación permiten al usuario moverse en un escenario a través de los avatares para poder interactuar con los entornos 3D, con acciones como caminar, volar, teletransportarse, editar terrenos, crear contenidos, manipular objetos virtuales, volar en forma libre y caminar [12]. Además, el manejo de colisiones hace más real el desplazamiento del avatar en el universo creado. La animación de los objetos virtuales integrados, permiten dar vida y mayor realismo a las propuestas, y al poder integrar sonido espacial, aumenta considerablemente esta sensación. Con las simulaciones físicas, se aumenta considerablemente el realismo de la interacción, logrando efectos de gravedad y fricción. También la posibilidad de integración de algoritmos desde la inteligencia artificial, permite aumentar las posibilidades de construir contextos simulados con múltiples aplicaciones [10].

Todas estas características mencionadas, han motivado en este proyecto diseñar e implementar un conjunto de escenarios inmersivos con diferentes actividades centradas en los temas del currículo de Física Mecánica, con el fin de observar y documentar el comportamiento y reacciones de los estudiantes de Ingeniería al utilizar esta tecnología y así lograr identificar la utilidad pedagógica en la aplicación de este tipo de tecnologías a procesos de enseñanza.

Algunas de las características de interacción diseñadas se han centrado en la propuesta de varios escenarios interconectados a través de caminos, simulando la estructura de un mapa conceptual de la asignatura de Física Mecánica. El concepto de exploración y acceso a las actividades, está soportado bajo algunas mecánicas de juego básicas, para promover el logro de los retos, avance de niveles y adquisición de puntajes requeridos para la exploración.

\section{Estado del arte}

La aplicación del concepto de los metaversos en el ámbito educativo, ha generado el desarrollo de diversos proyectos tanto a nivel nacional como internacional. Por ejemplo, eXtension ${ }^{\circledR}$ es una red educativa para el Sistema de extensión cooperativa 
de Land Grant Universities en los Estados Unidos [13].

El campus de la Universidad de Rutgers posee 34 regiones, que incluye un área principal de bienvenida, tiendas, espacios de exhibición de arte y muchas reproducciones de las edificaciones del campus universitario. Incluso ofrecen una administración del mundo virtual para crear los propios entornos virtuales para enseñar, explorar, y agregar cualquier cosa al curso [14].

El campus virtual de la Universidad de Tulane es usado en cursos libres de economía con juegos de libre comercio y desafíos de monopolio y otras simulaciones y juegos de rol para clases de economía [15].

El Centro de Simulaciones y Ambientes Virtuales de la Universidad de Cincinnati desarrolla aplicaciones de tecnología de inmersión para la investigación, colaboración científica y educación superior. Ayudan en la investigación a resolver problemas del mundo real en espacios virtuales seguros, alentando a los profesores y administradores a re-imaginar la experiencia universitaria con la realidad virtual y aumentada, y además se asocian con la industria para desarrollar visualizaciones de datos de forma innovadora, simulaciones de entrenamiento y experiencias de usuario [16].

La Universidad de Nueva Inglaterra está implementando un mundo virtual basado en OpenSim para estudiantes de farmacia y trabajo social, con el proyecto SPICE, para la contextualización de la experiencia de la interacción en el trabajo social en Farmacia [17].

A nivel nacional, la institución universitaria CESMAG de la ciudad de Pasto, desarrolló un entorno virtual 3D para apoyar procesos de aprendizaje en el curso de "Teoría general de sistemas" del programa de Ingeniería de Sistemas, en donde buscaron identificar los impactos y evidenciaron resultados significativos en cuanto a la apropiación de conocimientos por parte de los estudiantes [3].

En la Universidad Distrital Francisco José de Caldas, el grupo de investigación GICOGE implementó un metaverso denominado "SUA”, buscando apoyar procesos de aprendizaje en las asignaturas de programación básica y programación orientada a objetos de la Facultad de Ingeniería, logrando identificar resultados de participación notablemente mayores en un curso con respecto a otro con condiciones diferentes [18].

Otro caso ha sido el proyecto de la corporación Uniminuto de Bogotá quienes realizaron un prototipo de campus virtual, denominado Innova-T3D en donde crearon actividades para el aprendizaje de la unidad temática de Netiqueta, correspondiente al curso de Gestión Básica de la Información. Se observó que los niveles de participación y motivación en el grupo experimental fueron más altos que en un grupo de control [19].

\section{Metodología de investigación}

La puesta en marcha del presente proyecto, implicó el planteamiento de dos fases en la metodología de investigación. Se determinó en la primera, la realización de un análisis con carácter exploratorio, con el cual se buscó aumentar el nivel de familiarización con fenómenos relativamente desconocidos, analizando el comportamiento y reacciones de los estudiantes ante la aplicación de una tecnología relativamente nueva, por el uso de metaversos a procesos educativos en la Universidad Militar Nueva
Granada y con un enfoque gamificado. La gamificación o ludificación es un término que describe la aplicación de dinámicas del juego a cosas que en principio no lo son [20], como la obtención de puntos, medallas, insignias, pasar niveles, cumplir retos, entre otros. En una segunda fase, se ha planteado un trabajo relacional o explicativo, buscando analizar las variables de los niveles de motivación en los aprendizajes de los temas de la materia en cuestión y el porcentaje de pérdida de Física Mecánica.

El desarrollo de la prueba piloto fue aplicado a dos grupos de 30 estudiantes de segundo semestre de la Facultad de Ingeniería. Además, con el interés de obtener resultados en el estudio con la participación de los estudiantes y que hubiese un nivel inicial de motivación, se acordó que el trabajo realizado por ellos, tuviera una participación de un $20 \%$ en la nota formal de la asignatura.

\section{Metodología para la construcción del metaverso}

El diseño y puesta en marcha del mundo virtual, ha implicado la estructuración de una serie de fases, las cuales se mencionan a continuación.

\subsection{Fase 0: Condiciones iniciales}

Las condiciones iniciales para el modelo virtual del metaverso, han permitido mantener una idea clara de lo que se buscaba con el proyecto, y orientaron los lineamientos de diseño instruccional y el proceso de modelamiento de los diversos escenarios. Las siguientes condiciones fueron las que se tuvieron en cuenta:

- No pretender replicar los laboratorios físicos de la universidad.

- No ser un repositorio de extensos documentos o lecturas.

- No utilizar las mismas herramientas que se puedan articular en una plataforma LMS, como talleres, foros, encuestas, entre otras.

- Plantear un modelo virtual atractivo y moderno que despierte interés en los estudiantes, buscando un ingreso frecuente.

- Aplicar el mapa conceptual de la asignatura como una estructura de navegación.

Tomando como base lo anterior, se propuso realizar un modelo de laboratorios semejante a una red de conceptos, en la cual el estudiante pudiese viajar al interior de los mismos a partir de lineamientos gamificados, como la recolección de puntos para abrir compuertas y tener acceso a los diversos escenarios. El primero de ellos, fue el de ayuda o tutorial en el cual los estudiantes debían conocer las acciones y comandos desde el teclado y el ratón que pueden aplicar para navegar más eficientemente [12].

\subsection{Fase 1: Identificación de los temas de estudio}

La primera fase del proyecto implicó el desarrollo de un mapa conceptual de la asignatura, en donde se identificaron los temas principales de la materia y la relación entre ellos como se muestra en la Fig. 2. Se identificaron 8 escenarios en: “metrología”, “vectores”, “cinemática”, “movimiento relativo”, “dinámica”, "trabajo y energía”, "momento lineal y colisiones” y finalmente "centro de masa”. 


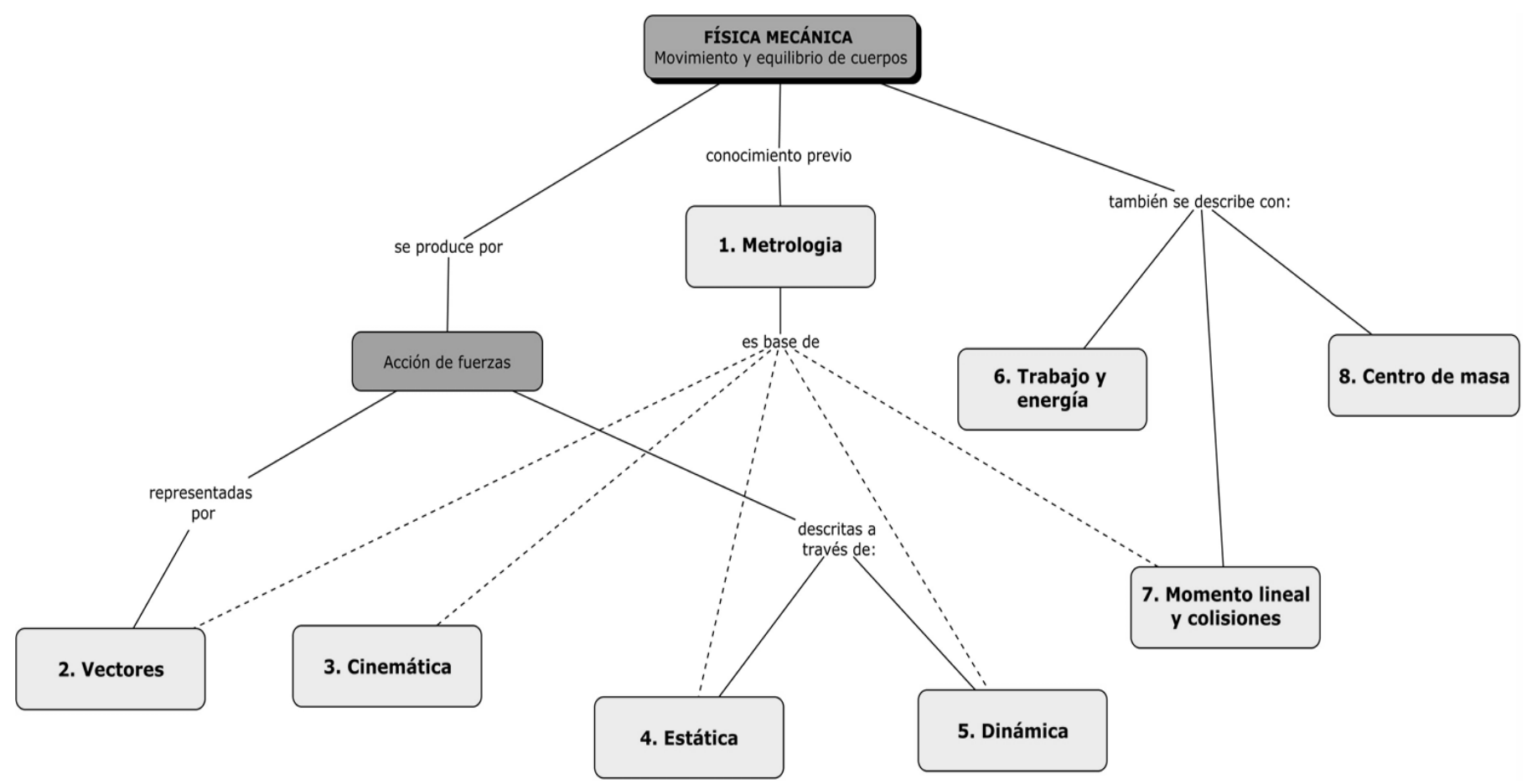

Figura 2. Mapa de conceptos de la asignatura.

Fuente: Los autores.

Tabla 1

Principales temas de la asignatura.

\begin{tabular}{ccc}
\hline Corte evaluativo & Unidad & Temas a estudiar \\
\hline \multirow{2}{*}{ Primer nivel } & 1 & Metrología \\
& 2 & Vectores \\
& 3 & Cinemática \\
\hline \multirow{2}{*}{ Segundo nivel } & 4 & Movimiento Relativo \\
& 5 & Dinámica \\
\hline \multirow{2}{*}{ Tercer nivel } & 6 & Trabajo y Energía \\
& 8 & Momento Lineal y Colisiones \\
& 8 & Centro de masa \\
\hline
\end{tabular}

Fuente: Los autores.

En la Tabla 1, se observa la distribución de los temas organizados en 3 niveles de acuerdo a los cortes evaluativos establecidos por la universidad.

\subsection{Fase 2: Diseño de las actividades y gamificación}

En esta fase se buscó identificar un concepto de la realidad asociado a cada tema, para poderlo abstraer y así modelar una propuesta gráficamente viable, buscando que el diseño de las actividades para cada uno de los temas, reforzaran la comprensión en la solución de problemas de Física Mecánica y poner en práctica la aplicación de los conceptos esenciales de la materia.

Antes de iniciar con la construcción y programación del metaverso, se realizó el diseño instruccional de lo que cada entorno debería contener, a partir de un formato de guion en el cuál se identificó el tipo de escenario, los medios de apoyo en imágenes, audio, video, ejercicios de aplicación, restricciones de acceso y componentes de gamificación, como las condiciones de obtención de insignias que recompensa la finalización de todas las actividades del metaverso, las cuales representan puntos extras, reflejados en la nota obtenida y en la tabla de posiciones del aula virtual.

Se caracterizaron entonces cada uno de los escenarios a utilizar, presentando a continuación, una breve descripción de cada uno de ellos.

Metrología: Se propuso un laboratorio con un ambiente futurista, en el cual el estudiante pudiese interactuar con diferentes objetos distribuidos en el escenario, los cuales presentan conceptos fundamentales necesarios para comprender la ciencia de la medición.

Vectores: Se propuso un laberinto que permitiera evidenciar una de las tantas aplicaciones vectoriales en la vida cotidiana. Allí el estudiante debía recorrerlo para poder llegar al siguiente nivel, y para evitar perderse en el recorrido, debía resolver una serie de preguntas que lo ubicaban espacialmente y así llegar a la salida en la ruta más corta.

Cinemática: En un escenario desértico de una pista de carreras de camionetas, el estudiante debía aplicar el conocimiento en las clases de movimientos para poder atravesar toda la pista superando diversos obstáculos. En las rectas, subidas y bajadas, los estudiantes debían asociar la aplicación de movimientos rectilíneos y para llegar a la meta, en un último tramo donde no había carretera, se tenía que aplicar conceptos de movimiento parabólico.

Movimiento relativo: Para este escenario se propuso una galería de imágenes, donde cada una representaba un concepto relacionado con el tema. Allí el estudiante debía asociar cada imagen con su respectiva definición, buscando reforzar el aprendizaje basado en la observación e identificación de imágenes para entender cada mensaje transmitido, debido a que 
los estudiantes tienden a ser más audiovisuales.

Dinámica: Se desarrolla aquí una pista de obstáculos en un parque con superficies lizas y de rozamiento, en donde se debe arrastrar un baúl de $40 \mathrm{~kg}$. Para lograr llegar a la meta, hay que conocer y comprender las leyes de Newton, puesto que, para trasladar la caja, se tenían que aplicar fuerzas diferentes y así tener que interpretar los tipos de fuerzas que actúan en un sistema.

Trabajo y energía: El escenario pensado para este tema se relaciona con una montaña rusa, ya que su funcionamiento se basa en el principio de la conservación de la energía. Aquí el estudiante se ubica al interior de una esfera y para llegar al final del trayecto, debe recorrer la montaña aplicando los conocimientos de las clases de energía mecánica, el principio de conservación de energía y el teorema de trabajo y energía. La montaña rusa está inmersa en el espacio, buscando generar una sensación de adrenalina, evitando ser lanzado hacia la nada.

Colisiones: Se propuso aquí un escenario simulando una biblioteca estilo café literario con cuentos didácticos de física, buscando fomentar la lectura divulgativa en Mecánica. Al terminar de leer el cuento escogido, los estudiantes deben completar una frase que hace parte de la lectura y así activar el indicador de respuesta correcta. Además, se proyecta un video de una clase que explica con ejemplos el tema, para lograr una mejor comprensión y mostrar a los estudiantes que hay otras formas para su aprendizaje.

Centro de masa: Por último, se desarrolló en un espacio con compuertas, en donde aparecen sistemas homogéneos y no homogéneos a los cuales se les debe calcular el centro de masa. $\mathrm{Al}$ acertar la respuesta, se abrirá una compuerta en el sistema y el estudiante podrá atravesarlo volando. Se planteó este escenario con el objetivo de que los estudiantes no solo aprendan a calcular el centro de masa, sino que además perciban gráficamente el lugar donde se supone debe estar concentrada toda la masa en los sistemas y cómo es su velocidad dependiendo de los cuerpos implícitos en éstos.

El resultado de esta fase se observa en el diagrama de la Fig. 3 , en donde se presenta el flujo de acceso a cada uno de los escenarios con las respectivas características de cada entorno, el objetivo y la recompensa obtenida.
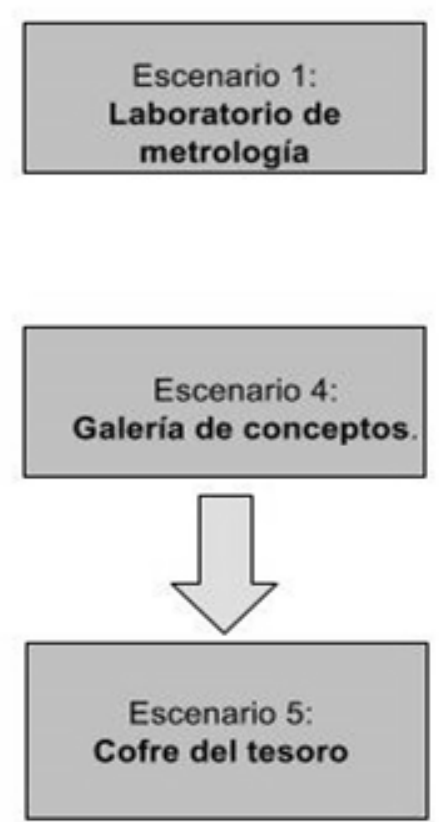

Escenario 8:

Recorrido con compuertas

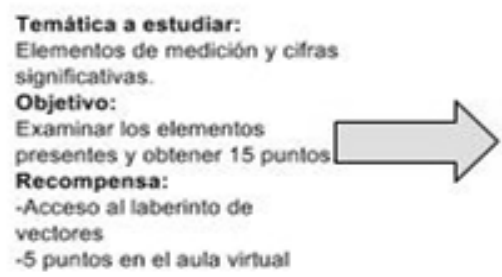

Temática a estudiar: Conceptos relacionados con movimiento rolativo. Objetivo:

Examinar los elementos de la galeria y obtener la clave de acceso a la pruoba de conocimientos

Recompensa:

Acceso al colre del tesoro

-5 Puntos en el aula virtual

Tematica a estudiar: Leyes de Newton. Objetivo:

Abrir el colte al final del recorrido para obtener la clave

Recompensa:

Acceso a la montan̂a rusa - Clave de acceso a prueba de conocimientos.

-15 puntos en el aula virtual

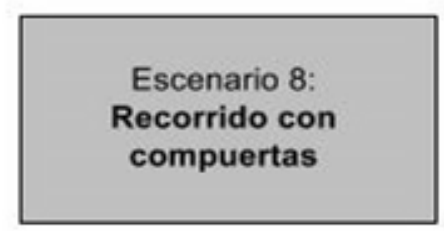

Temática a estudiar: Centro de masa Objetivo:

Atravesar todas las compuertas para llegar al fina del escenario

Recompensa:

Finalización de todas las

actividades del entorno

virtual.

-10 puntos en el aula virtual

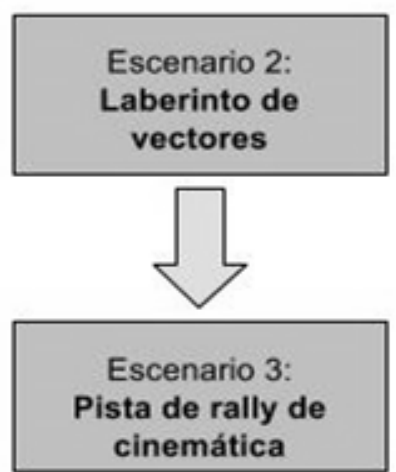

Temática a estudiar:

Operaciones con vectores Objetivo:

Encontrar la salida del laberinto

Recompensa:

-Acceso a la pista de rally de cinemática

10 puntos en el aula virtual

Temática a estudiar:

Ecuaciones de movimiento Objetivo:

Llevar la camioneta hasta el final de la pista.

Recompensa:

-Acceso a la galeria de conceptos de movimiento relativo

15 puntos en el aula virtual
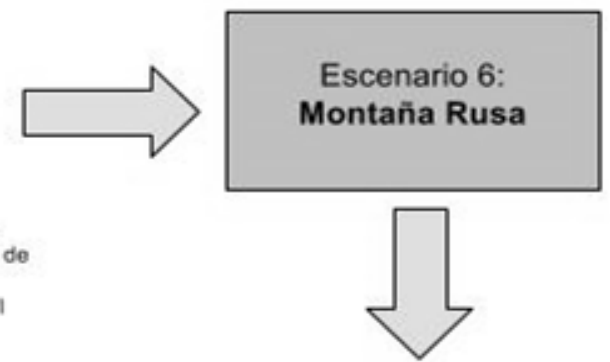

Escenario 7:

Café Literario
Tematica a estudiar: Trabajo y energia, conservación de la energia. Objetivo: Llevar la camioneta hasta el fina! de la pista. Recompensa:

Acceso a la galería de conceptos de movimiento relativo -20 puntos en el aula virtual

Temática a estudiar: Cantidad de movimiento lineal y colisiones Objetivo:

Leer todas las lecturas y responder correctamente las preguntas.

Recompensa:

-Acceso al nivel final, recorrido con compuertas.

-20 puntos en el aula virtual

Figura 3. Conceptos para escenarios del metaverso.

Fuente: Los autores. 


\subsection{Fase 3: Instalación y alojamiento en servidor}

En esta fase se realiza la instalación de la plataforma virtual OpenSim en un servidor dispuesto por la universidad, con las características técnicas particulares apropiadas para su almacenamiento y ejecución. La versión utilizada fue "diva distro", debido a que ésta ofrece un módulo para el registro de cuentas en línea, además que su configuración es rápida e intuitiva.

Las características técnicas del servidor utilizado se muestran en la Tabla 2, junto con algunos datos de rendimiento con el aplicativo.

En la Fig. 4 se puede observar que los valores correspondientes a los cuadros por segundo en el metaverso (SimFPS) y en las físicas aplicadas (PhyFPS) son buenos, considerando que los valores de 30 y 60 FPS son los más utilizados en el renderizado de videojuegos y aplicaciones 3D, siendo $60 \mathrm{FPS}$ el valor que mayor fluidez presenta en pantalla [21,22].

\subsection{Fase 4: Proceso de modelado y texturizado}

Se procede a generar un terreno plano virtual en donde se ubicarían los diferentes escenarios, creando una "región virtual" de 1000x1000 metros. Luego se crearon progresivamente cada una de las islas que se ajustaban al modelo gráfico del mapa conceptual de la asignatura.

En cada isla se construyó el escenario propuesto, teniendo que colocar los objetos tridimensionales requeridos, los cuales fueron creados con un bajo nivel de polígonos mostrado en la Fig. 5, con el fin de proporcionar un funcionamiento óptimo una vez fueran cargados al servidor. Los objetos que requirieron un mayor nivel de detalle, se construyeron en Blender, software de modelado y animación, mientras que aquellos objetos que presentaran un bajo nivel de estructura y detalle, fueron diseñados directamente en las herramientas de modelado que ofrece el visor "Firestorm".

Tabla 2

Características del servidor.

\begin{tabular}{cc}
\hline Tipo de servidor & Físico \\
\hline Sistema Operativo & Linux Ubuntu \\
Memoria RAM & $4 \mathrm{~Gb}$ \\
Disco Duro & $50 \mathrm{~Gb}$ \\
Procesador & 8 Núcleos \\
& Apache \\
Servicios & MySQL \\
& Php \\
IP & Pública \\
\hline
\end{tabular}

Fuente: Los autores.

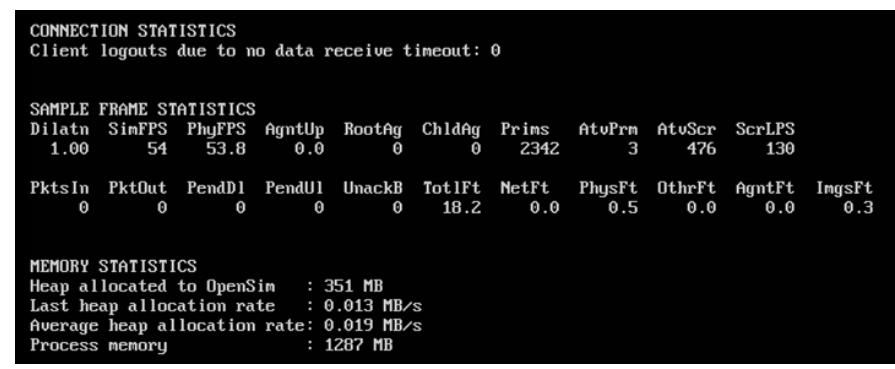

Figura 4. Estadísticas de ejecución del metaverso en el servidor.

Fuente: Los autores.

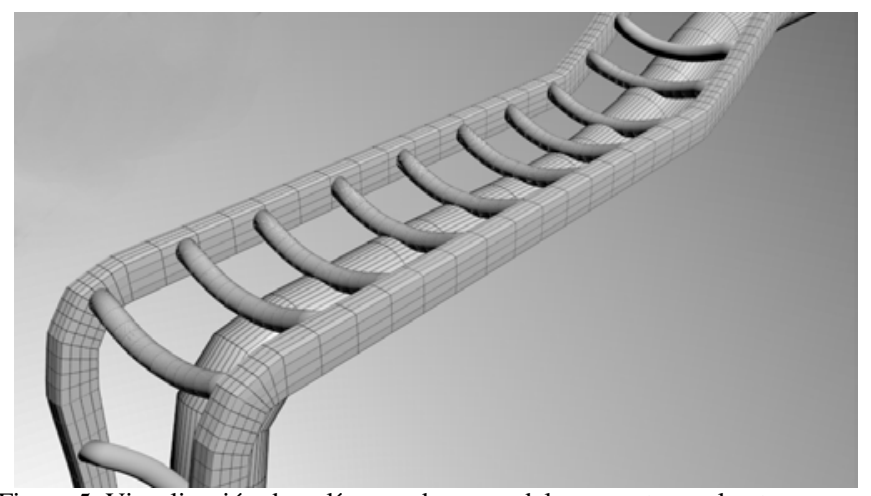

Figura 5. Visualización de polígonos de un modelo presente en el entorno. Fuente: Los autores.

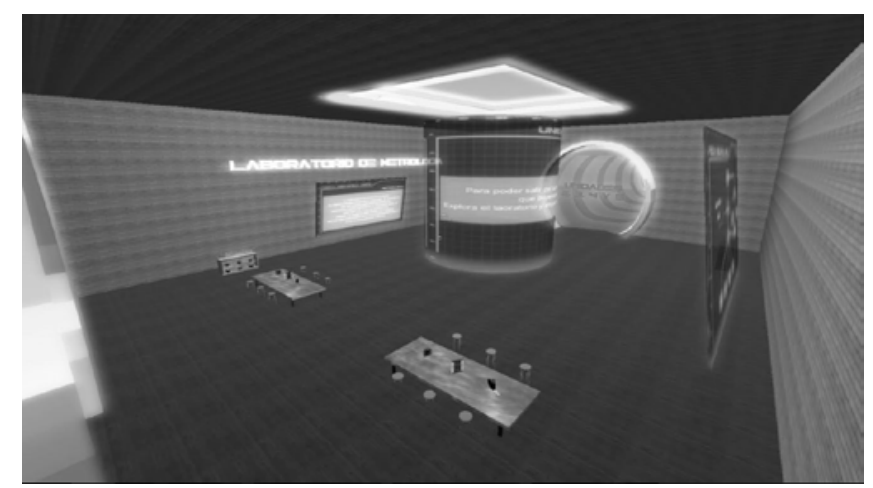

Figura 6. Escenario del metaverso para Metrología.

Fuente: Los autores.

Para el proceso de texturizado en la mayoría de los objetos poligonales, se utilizaron los componentes que ofrece la plataforma opensim, buscando que el renderizado en tiempo real, fuese el menos denso posible, pero para aquellos objetos con información puntual como los carteles de instrucciones, se diseñaron por medio de la herramienta de creación de gráficos vectoriales Inkscape.

En las siguientes imágenes, se muestran cada uno de los escenarios modelados, comenzando por el de metrología en la Fig.6.

El escenario construido para el tema de vectores se muestra en la Fig. 7.

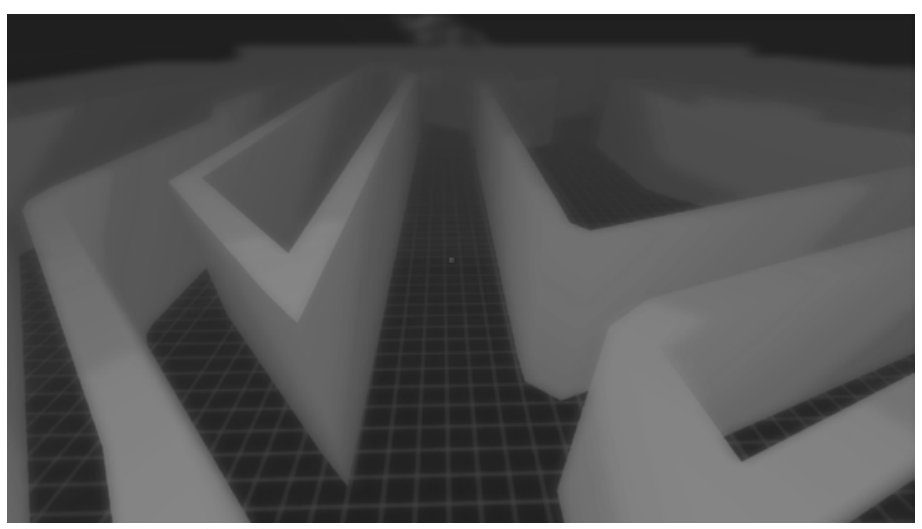

Figura 7. Escenario del metaverso para Vectores.

Fuente: Los autores. 


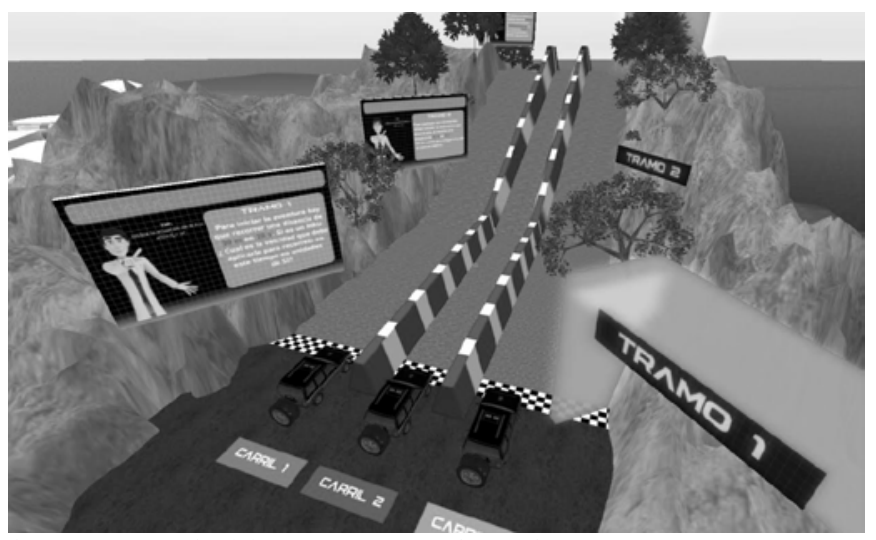

Figura 8. Escenario del metaverso para Cinemática.

Fuente: Los autores.

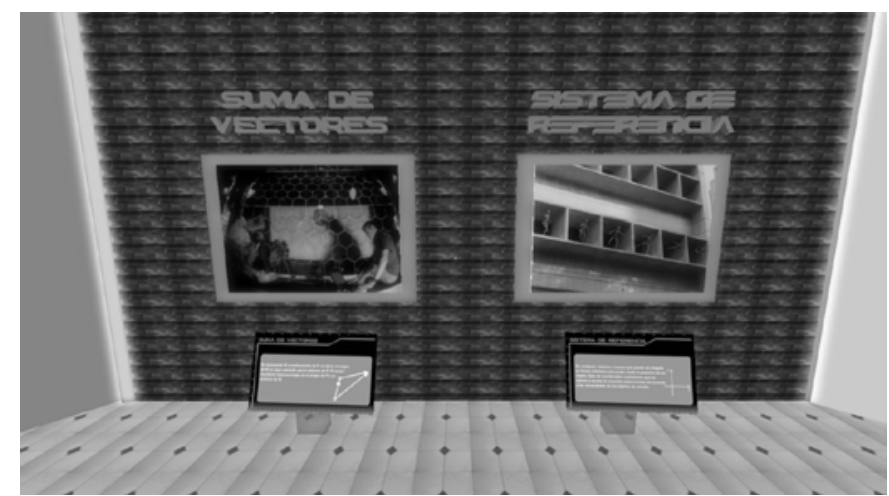

Figura 9. Escenario del metaverso para Movimiento Relativo. Fuente: Los autores.

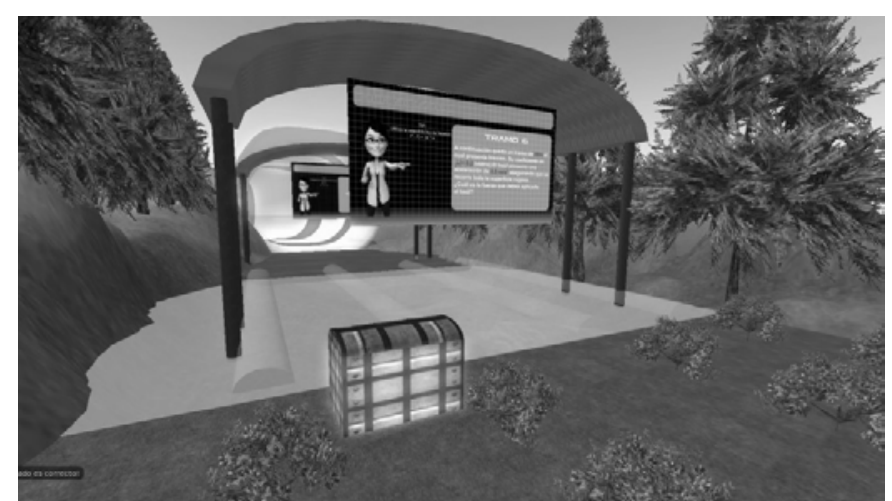

Figura 10. Escenario del metaverso para Dinámica.

Fuente: Los autores.

El modelo del escenario de cinemática se presenta en la Fig. 8. El escenario para movimiento relativo se muestra en la Fig. 9. Escenario para dinámica se muestra en la Fig. 10.

En la Fig. 11 se presenta el escenario para el tema de trabajo y energía. En la Fig. 12 presenta el escenario para el tema de colisiones.

Escenario para el centro de masa se presenta en la Fig. 13.

\subsection{Desarrollo de scripts en lenguaje LSL}

Los scripts (pequeños fragmentos de código en lenguaje de programación LSL - Linden Scripting Language asociados a los objetos presentes en el entorno), permitieron la interacción entre los usuarios y elementos en el entorno. Se tuvieron en cuenta las buenas prácticas de programación en este lenguaje, tales como la elaboración de códigos con pocas instrucciones específicas en lugar de scripts muy largos con gran cantidad de instrucciones, debido que a nivel general resultan más estables y efectivos.

Como el lenguaje LSL no permite la programación orientada a objetos (POO), se optó por un enfoque basado en funciones y máquinas de estado en la elaboración del código. Además, se tuvo en cuenta una característica muy importante que ofrece este lenguaje, que consiste en la comunicación entre scripts. Elementos como las camionetas, baúles, inclusive los "roller-ball” de la montaña rusa hacen uso de esta característica.

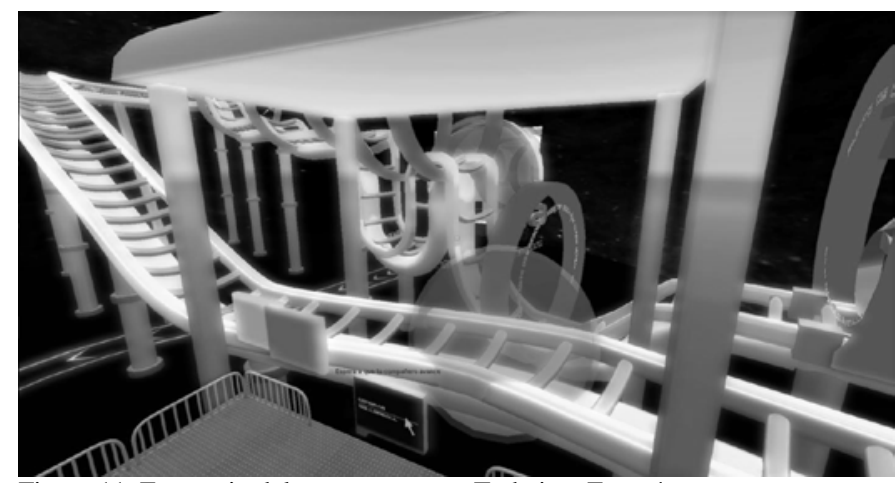

Figura 11. Escenario del metaverso para Trabajo y Energía.

Fuente: Los autores.

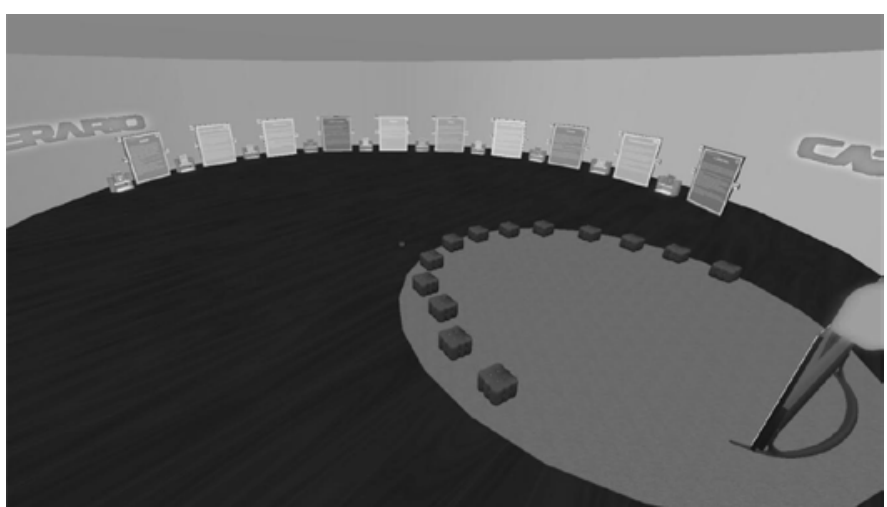

Figura 12. Escenario del metaverso para Colisiones.

Fuente: Los autores.

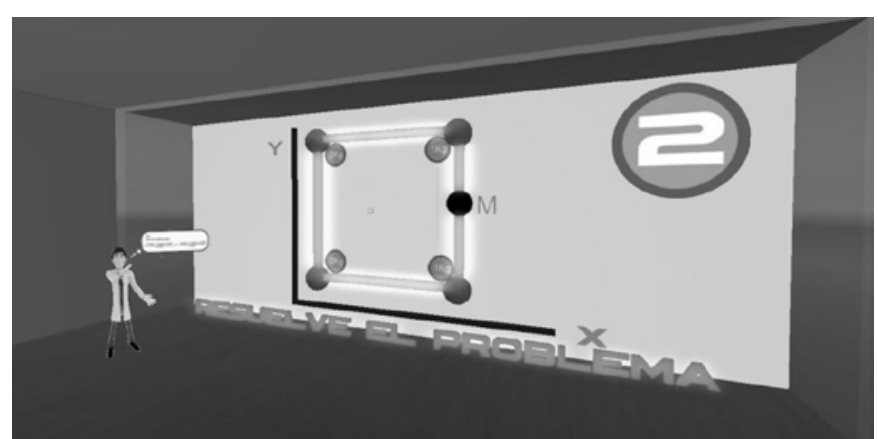

Figura 13. Escenario del metaverso para Centro de Masa.

Fuente: Los autores. 


\section{Resultados y discusión}

De manera complementaria al proyecto, se dispuso un aula virtual en Moodle para que los estudiantes mostraran las evidencias del desarrollo de las actividades propuestas en el metaverso, a través de una captura de pantalla en determinados puntos claves del escenario.

A continuación, se presentan los resultados obtenidos. En la Fig. 14 se evidencia el número de estudiantes que terminaron las actividades correspondientes en cada escenario. Como se observa, para el primer nivel, fue relativamente bajo, sin embargo, se incrementó notablemente el índice de finalización en los escenarios 4 y 5 , correspondientes a la galería de conceptos y el cofre del tesoro.

Con el objetivo de incrementar el acceso y finalización de las actividades del entorno 3D, se acordó con el docente de la asignatura, que algunas preguntas planteadas en el aula virtual, serían tenidas en cuenta en el examen parcial del segundo corte evaluativo. Se propuso la idea de incluir contraseñas de acceso a las pruebas de conocimiento en el aula virtual, las cuales debían ser obtenidas al finalizar los retos planteados. Como consecuencia, se puede evidenciar que el número de estudiantes que finalizaron las actividades en el metaverso, se incrementó en un 30\% aproximadamente.

Para el tercer corte se mantuvo el acuerdo planteado con el docente, sin embargo, no se generó una conexión directa entre el entorno 3D y el aula virtual por medio de contraseñas; lo cual afectó la culminación de las actividades, observando que en el escenario 6 el número de estudiantes que finalizó esta actividad fue similar a los registrados en el primer nivel.

Ya en el escenario 7 se presentó un incremento debido a las características propias de la actividad, permitiendo inferir que los estudiantes prefieren desarrollar actividades de menor complejidad matemática, posiblemente por la rapidez y facilidad que representa el desarrollo de éstas.

Para el último escenario, la culminación de la actividad se vio afectada debido a que el tema propuesto no se alcanzó a desarrollar en su totalidad en la clase presencial, además la finalización del semestre académico influyó notablemente en el interés de los estudiantes.

Número de estudiantes que finalizaron las actividades del entorno 3d

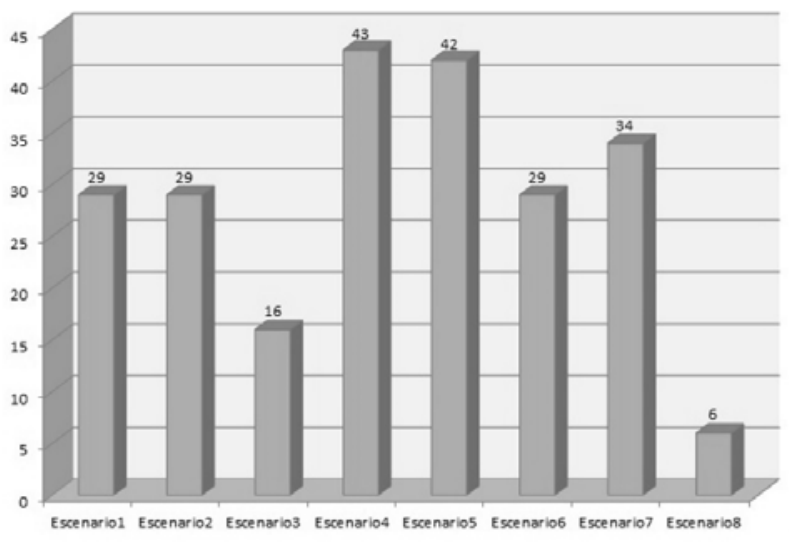

Figura 14. Número de estudiantes que finalizaron las actividades del metaverso. Fuente: Los autores.

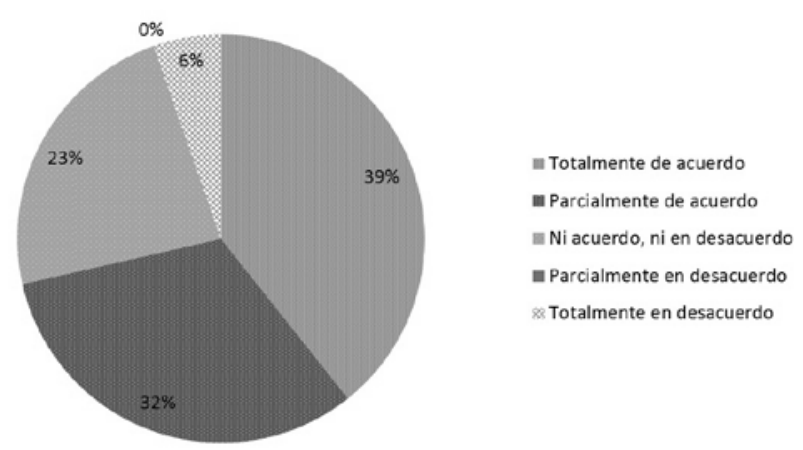

Figura 15. Porcentajes de motivación en la realización de las actividades del metaverso.

Fuente: Los autores.

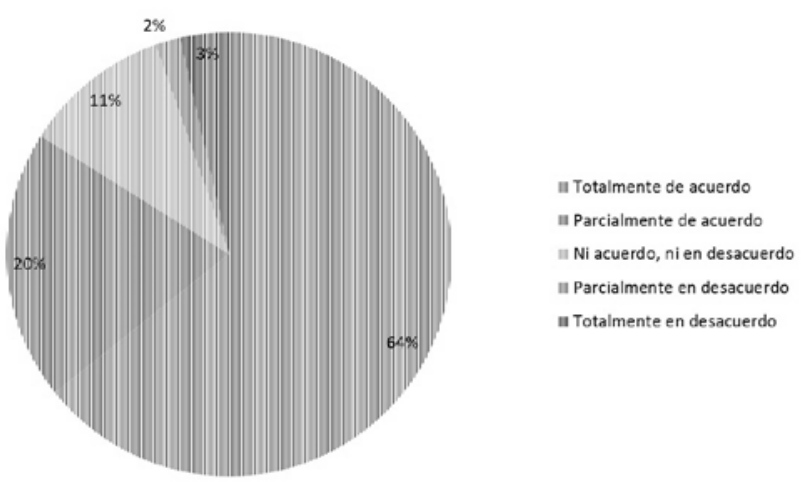

Figura 16. Porcentaje de aceptación en el uso de puntajes en las actividades. Fuente: Los autores.

Buscando evidenciar el impacto de la aplicación de mundos virtuales en el aprendizaje de los estudiantes, se aplicó una encuesta, para determinar el nivel de interacción, aceptación, problemas y otros factores asociados al uso del metaverso.

La Fig. 15 permite observar las respuestas de los estudiantes ante la pregunta de que si ellos consideran que los escenarios del espacio 3D puestos a disposición, motivaron la realización de los ejercicios planteados. La mayoría de ellos, el $71 \%$, manifestaron estar de acuerdo, lo cual indica que la dinámica en el uso de esta tecnología influyó positivamente en la motivación para el desarrollo de las actividades.

En el siguiente cuestionamiento se plantea al estudiante que si cree que el método de acumulación de puntajes en las actividades, ayudó a motivar la realización de más actividades. La Fig. 16 deja ver el resultado.

Se observa claramente que la acumulación de puntajes, así como la obtención de logros influyó significativamente en el desarrollo de las actividades. Inclusive se presentaron casos en los que estudiantes manifestaron dudas acerca de cómo subir de posición en las tablas de posición del aula virtual, es decir que se sintieron motivados a ser los mejores, generándose competencia entre ellos.

Uno de los interrogantes clave aplicado en las encuestas fue acerca del impacto que este recurso generó en el aprendizaje de la asignatura. Esta es una forma de evaluar el desempeño y 


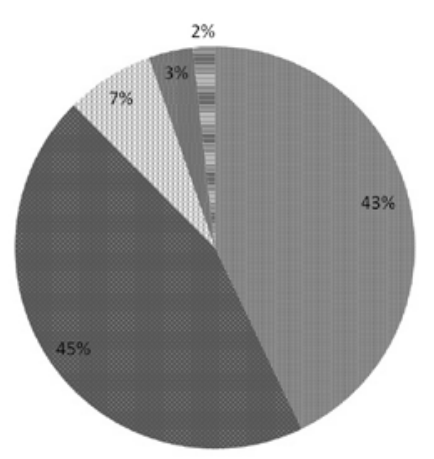

= Totalmente de acuerdo - Parcialmente de acuerdo In Ni acuerdo, ni en desacuerdo - Parcialmente en desacuerdo $=$ Totalmente en desacuerdo

Figura 17. Porcentajes de reconocimiento de que las actividades reforzaron el aprendizaje de la asignatura.

Fuente: Los autores.

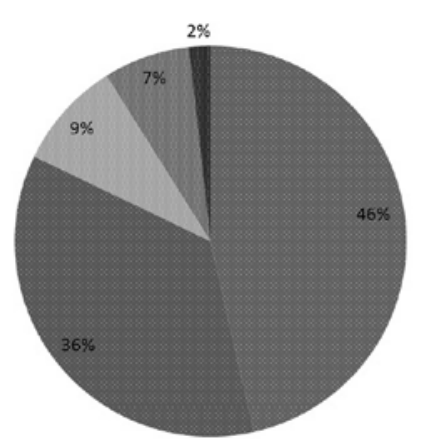

- Totalmente de acuerdo - Parcialmente de acuerdo $=$ Ni acuerdo, ni en desacuerdo w Parcialmente en desacuerdo - Totalmente en desacuerdo

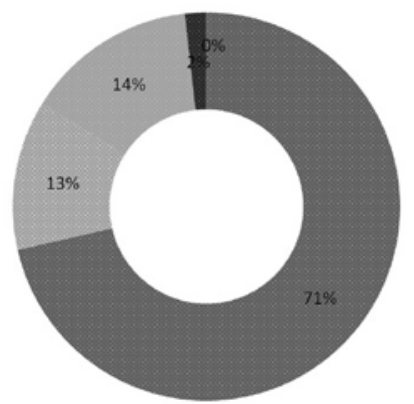

- Totalmente de acuerdo in Parcialmente de acuerdo $=$ Ni acuerdo, ni en desacuerdo m Parcialmente en desacuerdo - Totalmente en desacuerdo

Figura 19. Nivel de aceptación a recompensar en cierto porcentaje de la nota en la asignatura.

Fuente: Los autores.

efectividad en la aplicación de este tipo de tecnologías para potenciar el aprendizaje. Como se muestra en la Fig. 17, el 86\% de los estudiantes, manifestaron estar de acuerdo en que los temas estudiados fueron reforzados de alguna manera con el uso de los recursos puestos a disposición.

En la Fig. 18 se puede observar que el $82 \%$ de los estudiantes manifestaron estar de acuerdo en que el grado de motivación para aprender Física Mecánica, fue incrementado con el uso de estos recursos digitales.

Una de las condiciones que tuvo la aplicación de la prueba piloto, fue el acuerdo realizado con el docente a cargo de la

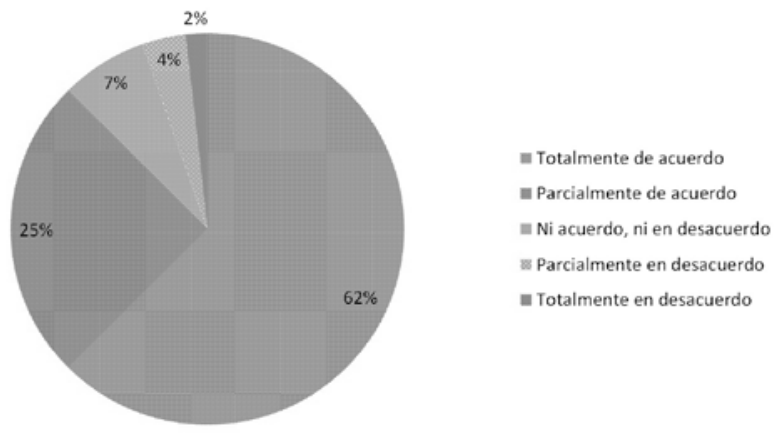

Figura 20. Nivel de satisfacción generado en los usuarios con la implementación del sistema de insignias.

Fuente: Los autores.

asignatura, quien facilitó un $20 \%$ a las actividades relacionadas con este proyecto en la nota de cada corte evaluativo.

De acuerdo con la Fig. 19, se puede observar que el uso de los recursos se vio fuertemente ligado a este factor, ya que la gran mayoría de los participantes estuvieron totalmente de acuerdo en que se otorgara un porcentaje de calificación a estas actividades. A pesar de que fueron complementarias a la asignatura, muchos de los estudiantes manifestaron haber utilizado los recursos debido a que sintieron curiosidad, además de querer complementar sus conocimientos de una manera alternativa.

Por otra parte, se quiso evaluar el efecto generado debido a la implementación de mecánicas de juego en el metaverso y el aula virtual, cuestionando la satisfacción en la obtención de insignias, obteniendo los siguientes resultados mostrados en la Fig. 20.

Se puede observar que la motivación en el desarrollo de las actividades se vio potenciada por la implementación de un sistema de insignias en el aula virtual. Razón por la cual se puede suponer que este tipo de mecánicas aplicadas en contextos educativos influyen positivamente en el aprendizaje de los estudiantes. Un 62\% estuvo totalmente de acuerdo con este planteamiento, al cual le podríamos sumar un $25 \%$ adicional que estuvieron parcialmente de acuerdo.

Finalmente se indagó a los usuarios acerca de si recomendarían el uso de este tipo de estrategias tecnológicas para apoyar procesos de aprendizaje a nuevos estudiantes como apoyo a las clases presenciales. Se observa en la Fig. 21, que un porcentaje bastante alto $91 \%$, estuvo de acuerdo, lo cual sugiere que en términos generales la aplicación de estas tecnologías fue satisfactoria.

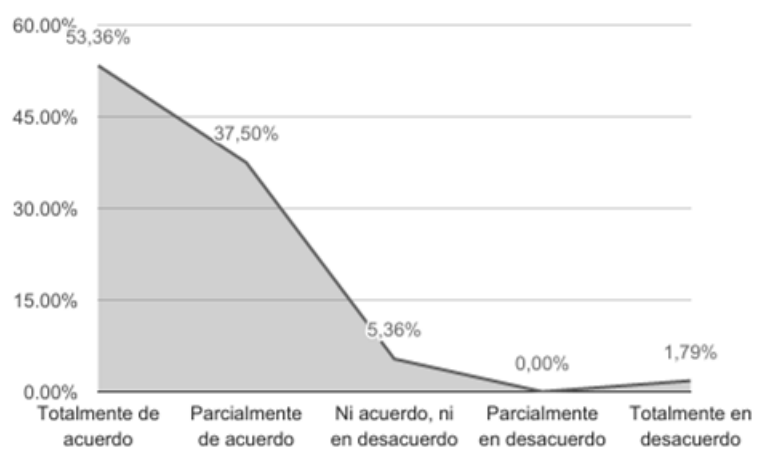

Figura 21. Porcentajes de posible recomendación al uso de los recursos por parte de usuarios.

Fuente: Los autores. 


\section{Conclusiones}

La aplicación de conceptos de gamificación a escenarios virtuales orientados al aprendizaje, conlleva a los diseñadores y docentes plantear de manera diferente las actividades, evitando el ofrecer recompensas sin ningún objetivo. Se deben medir y validar en forma permanente, las mecánicas de juego aplicadas e intentar detectar cuáles de ellas producen mejores resultados y reforzarlas. El uso de avatares que guíen y presenten los contenidos en estos escenarios es muy importante, ya que, a través de ellos, el docente puede expresarse de manera más cercana para intentar motivar al estudiante.

Los resultados obtenidos durante el primer corte evaluativo en la aplicación del piloto, hace considerar que se debe tener presente en el diseño de productos o recursos destinados a usuarios promedio, aspectos relacionados con la previsión. En este caso particular, la gran mayoría de los estudiantes hacen uso de tecnología diariamente, sin embargo, esto no aseguró que ellos comprendieran el manejo y configuración de las herramientas necesarias para la ejecución de este tipo de entornos, presentándose inconvenientes al inicio de la prueba piloto, en muchos de los estudiantes que deseaban acceder al entorno 3D pero no pudieron lograrlo por su cuenta.

El haber incluido escenarios virtuales como apoyo de aprendizaje en la asignatura de Física Mecánica, motivó a los estudiantes a enfrentarse a la solución de situaciones acorde al contenido programático de la materia, los cuales fueron diseñados con el objetivo que los estudiantes estuvieran inmersos en entornos en los que les facilitara asociar su aplicación con el mundo real, por medio de la interacción entre el avatar y el medio, permitiéndoles repasar y reforzar los conocimientos vistos en clase.

Es necesario resaltar la novedad en la implementación de los entornos 3D para Ciencias Básicas en la Universidad Militar Nueva Granada, donde con un pequeño tamaño muestral de los estudiantes de la Facultad de Ingeniería, se accede por primera vez a esta tecnología y a pesar del corto tiempo que se tuvo para familiarizarse con entornos 3D, se realizaron las actividades y presentaron las evidencias en la finalización de cada una de ellas. Los resultados permiten evidenciar que los estudiantes están abiertos a utilizar otras estrategias de aprendizaje apoyadas con tecnología, y así incentivar el desarrollo de metaversos en diferentes áreas del conocimiento.

Así también los estudiantes esperan que las instrucciones de las actividades que se planteen, sean más claras y detalladas, que no hubiese errores de cálculos en las operaciones y que fueran totalmente validados por el profesor directamente desde el entorno, ya que es factible cometer errores en el momento de las implementaciones.

Contar con vídeos explicativos de los ejercicios o más ayudas visuales para poder desarrollar con mayor atención las actividades, esperando de igual manera un mayor acompañamiento y retroalimentación del docente, ya que en el entorno virtual en ocasiones es difícil evidenciar los propios errores.

Finalmente, que hubiese una comunicación más directa entre el entorno 3D y el aula virtual, para que la entrega de las evidencias en las actividades se realice automáticamente y no hayan pasos adicionales.
Se espera poder mejorar el modelo propuesto, para así afinar los detalles que generaron inconformidad en los estudiantes durante la aplicación de este piloto y así seguir recolectando datos para evaluar la efectividad de este proyecto a mediano y largo plazo.

\section{Agradecimientos}

Este artículo es resultado del proyecto de investigación: "Diseño y puesta en marcha de un escenario virtual inmersivo orientado a procesos educativos y soportado en la navegación conceptual de un contenido de estudio" con código INV-ING2126, financiado por la Vicerrectoría de Investigaciones de la Universidad Militar Nueva Granada. Bogotá - Colombia.

\section{Referencias}

[1] Cabrera-Medina, J.M., Sánchez-Medina, I.I. y Rojas-Rojas, F., Uso de objetos virtuales de aprendizaje OVAs como estrategia de enseñanza aprendizaje inclusivo y complementario a los cursos teóricos - prácticos. Una experiencia con estudiantes del curso física de ondas, Revista Educación en Ingeniería., 11(22), pp. 4-12, 2016.

[2] Jiménez-García, F.N., Márquez-Narváez, C., Agudelo-Calle, J.D.J. y Beleño-Montagut, L., Una experiencia didáctica en el diseño e implementación de objetos de aprendizaje para la enseñanza de la física, Revista Educación en Ingeniería 11(22), pp. 13-20, 2016.

[3] Jiménez-Toledo, J.A., Muñoz-Botina, J.M. y Muñoz-del Castillo, A., Modelo virtual inmersivo 3D como estrategia didáctica en la educación, 2013.

[4] Diosa-Ochoa, Y., Enseñanza-Aprendizaje de la cinemática lineal en su representación gráfica bajo un enfoque constructivista: Ensayo en el grado décimo de la Institución Educativa Pbro. Juan J. Escobar, 2012.

[5] Dionisio, J.D., Burns, W. y Gilbert, R., 3D Virtual worlds and the metaverse: Current status and future possibilities, ACM Computing Surveys, 45(3), 2013. DOI: 10.1145/2480741.2480751

[6] Schank, R.C., Berman, T.R. and Macpherson, K.A., Learning by doing. Instructional Design Theories and Models, 2, pp. 161-181, 1999.

[7] Criado, M.A.P. y Thous-Tuset, M. Del C., Mundos virtuales y avatares como nuevas formas educativas, Historia y Comunicación Social, 18, pp. 469-479, 2013. DOI: 10.5209/rev_HICS.2013.v18.44262

[8] Castronova, E., Virtual worlds: A first-hand account of market and society on the cyberian frontier, CESifo Working Paper Series, Vol. 2, 2001.

[9] Checa-García, F., El uso de metaversos en el mundo educativo: Gestionando conocimiento en Second Life, REDU - Revista de Docencia Universitaria, 8(2), pp. 147-159, 2011.

[10] Ramos-Nava, M. del C., Larios-Delgado, J., Cervantes-Cabrera, D. y Leriche-Vázquez, R., Creación de ambientes virtuales inmersos con software libre. Revista Digital Universitaria [en línea]. 8(6), 2007. [Consultada: 11 de junio de 2007]. Disponible en: http://www.revista.unam.mx/vol.8/num6/art47/int47.htm

[11] Admin OpenSim, Análisis 02: OpenSim en la educación, 27 de abril de 2011. [Online]. Available at: http://o3dsoft.com/blog/es/2011/04/analisis-02-opensim-en-laeducacion/.

[12] Allison, C. et al., Growing the use of virtual worlds in education: An opensim perspective, EiED 2012 Proc. 2nd Eur. Immersive Educ. Summit, pp. 1-13, 2012.

[13] extension. [Online]. Available at: https://extension.org/2011/09/07/virtual-world-news/. [10-Feb-2017].

[14] Rutgers continuing Studies virtual worlds. [Online]. [Date of reference: 10-Feb-2017]. Available https://onlinelearning.rutgers.edu/virtualworlds.

[15] Tulane SCS Metaverse. [Online]. [Date of reference: 10-Feb-2017]. Available at: http://scsmetaverse.wp.tulane.edu.

[16] U. of Cincinnati, Center for Simulations \& Virtual Environments 
Research. [Online]. [Date of reference: 10-Feb-2017]. Available at: http://ucsim.uc.edu/blog/.

[17] University of New England, SPICE Social work Pharmacy Interaction Contextualization Experience. [Online]. [Date of reference: 10-Feb2017]. Available https://daneelariantho.wordpress.com/2011/10/16/spice/.

[10-Feb2017].

[18] Coronado, P.C., SUA: Metaverso especializado para el aprendizaje, en experiencias educativas y prácticas pedagógicas en la Universidad Distrital, 2013, pp. 123-132.

[19] Quinche, J.C. y González, F.L., Entornos virtuales 3D, alternativa pedagógica para el fomento del aprendizaje colaborativo y gestión del conocimiento en UNIMINUTO, Formación Universitaria, 4(2), pp. 4554, 2011. DOI: $10.4067 /$ S0718-50062011000200006

[20] Reig, D. y Vílchez, L.F., Los jóvenes en la era de la hiperconectividad: tendencias, claves y miradas, Fundación Telefónica. 2013.

[21] Gregory, J., Game engine architecture. Wellesley, Massachusetts: Taylor and Francis Group, LLC, 2009.

[22] Bithell, M., Understanding the importance of frame rate - IGN. [Online]. [Date of reference: 28-Feb-2017]. Available at: http://www.ign.com/articles/2014/11/05/understanding-frame-rate-andits-importance.
J.A. Jaramillo-Mujica, recibe el título de Ing. de Sistemas de la Universidad Autónoma de Colombia en 1995, el de Esp. en Gerencia de Tecnología de la Universidad EAN en 1998. MSc. en Desarrollo de Aplicaciones Multimedia para WEB de la UOC España en 2004 y el título de MSc. en Educación de la Universidad Militar Nueva Granada, Colombia, en 2015. Es experto universitario en la implementación de proyectos e-learning de UTN Argentina y cursó el diplomado en ambientes virtuales de aprendizaje con la FUCN Colombia y el diplomado en uso y apropiación de las TIC en la UMNG. Fue director del Departamento de Producción Multimedia de educación a distancia en la Militar y asesor del Centro de Altos Estudios del DANE - CANDANE en temas de virtualización. Ha venido desempeñándose como docente investigador en el programa de Ingeniería en Multimedia en la Universidad Militar Nueva Granada, en la cual coordina la cátedra electiva de Multimedia Educativa y el semillero de investigación con el mismo nombre. Tiene más de 20 años de experiencia en docencia y en el diseño e implementación de la metodología educación a distancia en instituciones como el Centro de Estudios Aeronáuticos de la Aeronáutica Civil Colombiana y la Universidad Militar Nueva Granada. ORCID: 0000-0003-0054-3796

L.F Morales-Avella, recibe el título de Ing. en Multimedia de la Universidad Militar Nueva Granada de Bogotá, Colombia en 2015. Ha sido parte del semillero de Investigación en Multimedia Educativa desde el año 2013, en donde desarrolló su opción de grado. Ha participado en eventos institucionales, regionales y nacionales de investigación, entre éstos el "V Concurso de Investigación Formativa" organizado por la Universidad Militar Nueva Granada, donde presentó el mejor trabajo en su categoría. Actualmente se encuentra desempeñándose como Joven Investigador asociado a un proyecto de investigación financiado por la Vicerrectoría de Investigaciones de la Universidad Militar Nueva Granada, Colombia.

ORCID: 0000-0001-5784-5168

D.M. Coy-Mondragón, recibe el título de Físico de la Universidad Pedagógica y Tecnológica de Colombia en 2010 y el de MSc. en Ciencias - Física de la Universidad Nacional de Colombia en 2015. Desde el 2012 ha dedicado a la docencia universitaria en las diferentes áreas de la física para los programas de Ingeniería en la Universidad Pedagógica y Tecnológica Colombia y en la Universidad Militar Nueva Granada, Colombia. Actualmente se desempeña como asistente de investigación de un proyecto de investigación financiado por la Universidad Militar Nueva Granada enfocado en la implementación de un modelo educativo apoyado con la implementación de metaversos, aulas virtuales y objetos de aprendizaje para la asignatura de Física Mecánica. ORCID: 0000-0003-2071-066X 